Proceeding

\section{Degenerative scoliosis}

\section{Aim of presentation}

A. Increase the attention of Spine surgeons about the size of the problem is it pathological or a natural process).

B. Discuss the changes in the morphology and biomechanics of the Lumber degenerative Scoliosis.

C. Discuss the correlation between the Pathomorpholegic changes and Clinical presentations.

D. Highlight the different treatment options.

E. Discuss the different surgical techniques.

F. Present few cases.

\section{Pathology and patho-mechanics}

a. Side to side curvature of the spine after skeletal maturity.

b. It could be present at the adolescent age before skeletal maturity and continue through adult ages.

c. It could be a result of incompletely corrected deformity at adolescent age.

d. It may start at elder age without any deformity during adolescence.

e. Pathology and Patho-mechanics.

f. The main cause is asymmetric deterioration of the facet joints, like osteoarthritis of the other joints in the body.

g. The spine starts to shift to one side at which the deterioration of the facets is more advanced.

h. Hypertrophy and ossification of ligamentum flavum with narrowing of the spinal canal and root canal.

i. Neural canal and root canal compromise.

j. Pathology \& Patho-mechanics.

k. Secondary curves above and below the degenerative curve may be difficult to develop in cases with previous fixation at adolescent age.

1. Loss of normal Lordosis, Vertebral displacement in frontal and sagittal planes (degenerative spondylithesis).

$\mathrm{m}$. Vertebral Displacement in lateral direction Latreolythesis.

$\mathrm{n}$. Loss of balance in frontal and mainly the sagittal plane.

o. C2 PLUM LINE Displacement.

\section{Clinical presentation}

A. Stiffness of the back and lower back mainly.

B. Pain in lower back and buttocks.

C. Radicular pain with numbness in the legs and feet.

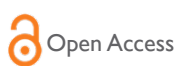

D) CrossMark
Volume 5 Issue 3 - 2016

Mohamed Maziad

Department of Orthopedics \& Spine Surgery, Ain shams University Cairo, Egypt

Correspondence: Mohamed Maziad, Department of Orthopedics \& Spine Surgery, Ain shams University Cairo, Egypt. Email maziad60@hotmail.com

Received: May 06, 2016 | Published: July 28, 2016
D. Motor and sensory weakness.

E. Muscular strain.

F. Progress of the curve 1-2\% increase in the degree per year

G. Loss of balance and development of compensatory deformities at other joints as $\uparrow$ hip extension and knee flexion.

H. Reduced normal dorsal kyphosis (Figure 1).

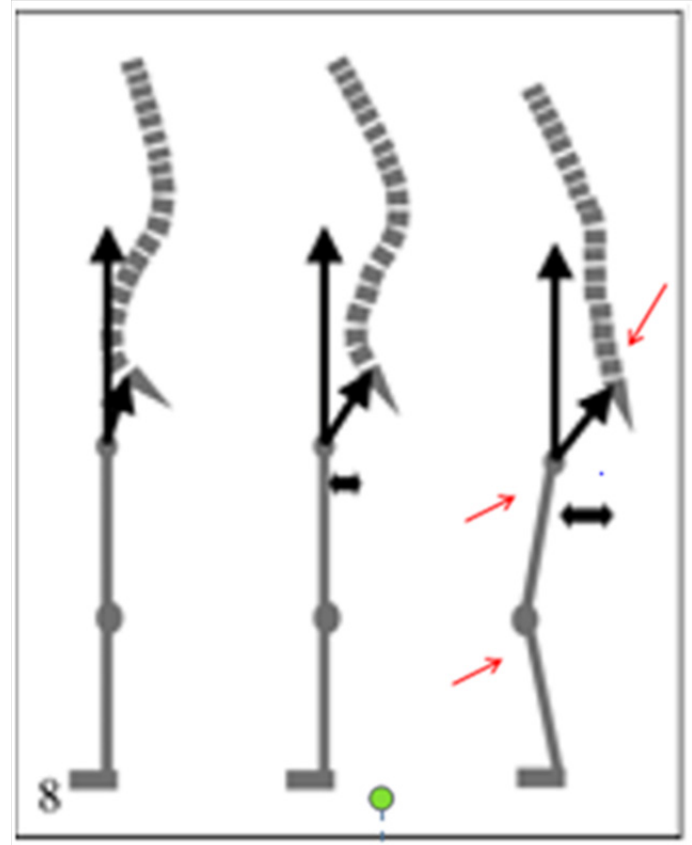

Figure I Compensation (Lumbar Kyphosis )à Decrease in S S $\uparrow$ Increase in PT, Hip Extension, Knee bend, Decrease in thoracic curve.

\section{Degenerative scoliosis is painful one}

a. Healthy facet joints acting like hinge that help spine pending smoothly. 
b. Aging naturally erode the cartilage that protect the joint.

c. Joints become irritable inflamed and painful with motions.

d. Disco genic pain as result of disc degeneration.

e. Neuro genic Claudication due to spinal canal stenosis.

f. Muscular spasm with pain as muscles tries to keep spinal alignment all the time.

\section{Nature of pain in degenerative scoliosis}

a. Gradually occurring and progressing pain.

b. Pain increasing with activities and movements.

c. It is worsen gradually by time.

d. Pain tends to be worse at the morning.

e. It improves by morning activities and getting worse after.

f. Setting feels better than standing and walking

g. Facets are less loaded in setting while facets loading increase in standing and walking.

\section{Degenerative scoliosis over the last I5yrs}

More patients with Degenerative scoliosis than before why?

Now People are living longer than before. Thy put more stresses on their spine with the increased daily activities. Patients became more demanding, less accepting the spine condition as an inevitable part of aging. Osteoporosis cause Degenerative scoliosis by the unequal vertebral compression and wedging fractures, loss Lordosis and increased normal kyphosis in the dorsal spine.

\section{Other symptoms associated with} degenerative scoliosis

a. Diffuse back pain over the curve on concave or convex side.

b. Pain at the bottom of the curve due to over loading of the last disc at L5-S1 Level.

c. Cloths become improperly fitting.

d. Patients become shorter by time as the curve progress. The rib cage seems to be fitting on the pelvis.

\section{Treatment options for patients with degenerative scoliosis}

Philosophy of treatment for patients with Degenerative Scoliosis.

I. Treatment should be directed to Pain Management.

II. Treatment for the compromised neural tissues.

III. Treatment of the Associated Osteoporosis.

IV. Correction of the curve is not the main concern of the treatment program, but restoration of the lost Balance calls for correction in certain cases.

\section{Tools for treatment}

I. Physiotherapy,Swimming Muscle building exercises.

II. Bracing.
III. Medical treatment for the Associated Medical problems.

IV. Care with respiratory functions.

V. Surgical interventions.

Every patient's condition should be studied very carefully; his surgery should be planned according to his own condition. Risk factors have to be weighed against the surgical benefits in order to achieve much better quality of life after major surgery at elder ages.

\section{Evaluation of patients with degenerative scoliosis}

a. Pain -type and sites of pain.

b. Neurological symptoms and signs.

c. Short breath easy becomes fatigued.

d. Cosmetic appearance Sites of the Curves.

e. Fitting of clothes.

f. General over view of patient health \& Medical problems.

g. Previous surgical correction and fixation.

h. Review of the old and recent $x$ rays for detection of curve progression.

i. Assessment of the old surgery.

\section{Conservative treatment program}

\section{A-Mild scoliosis less than $\mathbf{4 0}$ degrees}

Un-progressive curve treatment by

a. Exercises muscle building programs.

b. Aerobics, swimming, Maintaining of proper weight and proper natural Endorphin level by sport activities.

c. Pain killers and NAIDS are recommended with careful attention to their systemic effect especially on the kidney.

\section{B-Moderate scoliosis}

Bigger curves between (40-60) degrees. Balance is still maintained. Mild to less sever degrees of pain Treatment by:

A. TLSO Thoracic Lumbo-Sacral Orthotic -light weight.

B. Bracing for short periods takes the weights off the facets it is alternating with exercises.

C. Bracing cannot stop cure progression among elderly it is recommended for symptomatic relieve (Figure 2).

\section{C-The candidate for surgery}

a. Patients with major curves above 60 degrees or more.

b. Patients with clear evidences of curve progression by periodical examinations and radiological assessment.

c. Thoracic curves that casing pulmonary function deterioration.

d. Lumbar curves that causing neurological deficits.

e. Pain and other symptoms interfering with life quality. 

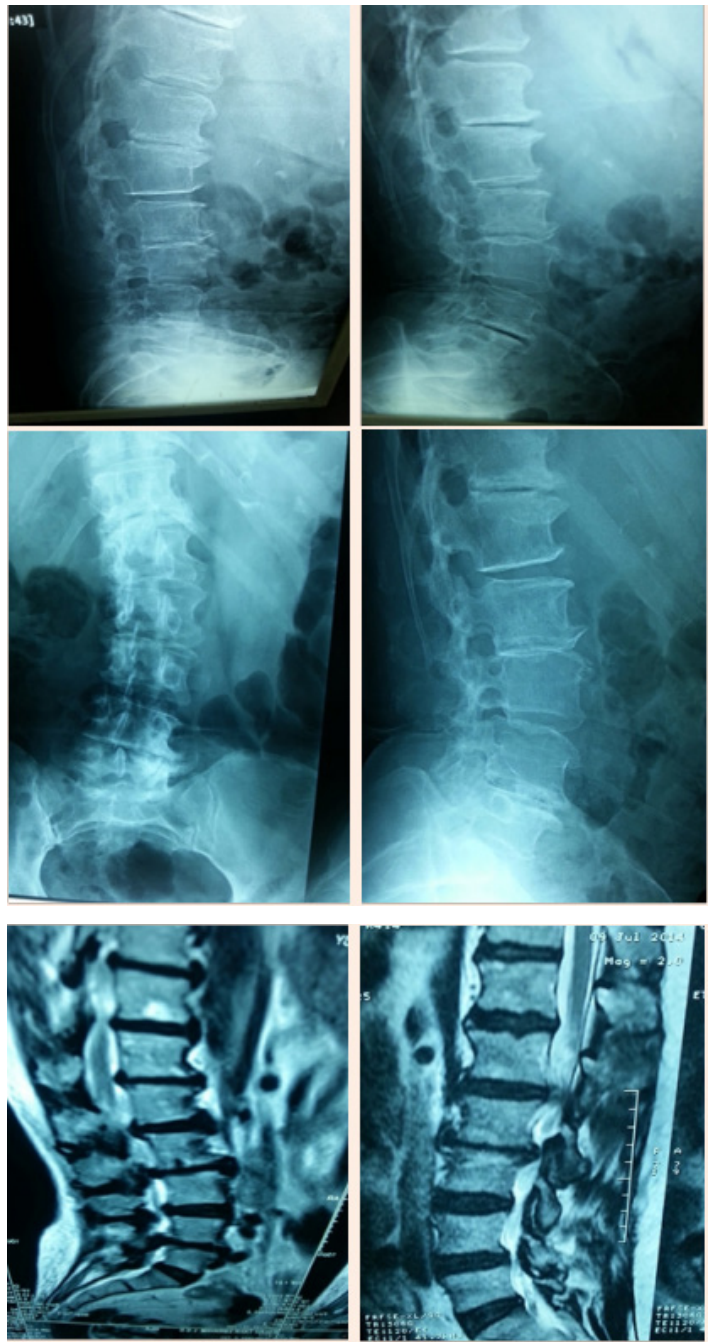

Figure 2 B-Moderate Scoliosis.

\section{Surgical planning and techniques}

a. Correction of Degenerative Scoliosis includes

b. Anterior and Posterior Surgery.

c. One Stage Surgery is more preferable.

d. Correction may carry high surgical risks.

e. Age is not the factor but the general health and biology.

f. Miss techniques both anterior and posterior became more popular and more adopted to reduce time and risks.

Uncorrective surgery with limited segments fusion within Balanced spine is a good alternative to full correction with long and extended fusion.

\section{Limited surgical interventions}

\section{Decompression surgery alone}

Limited intervention for:

a. Acute sequestrated disc within a localized segment in a scoliotec curve. b. Localized increase in segmental instability in balanced spine.

c. Minimally invasive Neuro spinal Endoscopic decompression.

d. It is not risky as open decompression, fusion and correction.

e. It could be repeated if needed after a period of pain cure.

\section{Open versus endoscopic decompression}

\section{Limited open decompression}

a. Limited open Decompression with inter spinal or inter- laminar devices.

b. Localized open fusion to certain motion segment.

c. Minimally invasive techniques for Anterior release, decompression and fusion through thoraco scopic interventions.

d. Minimally invasive tran's pedicular fixation have been practiced over the last decade with successful results.

e. AII of these techniques have reduced surgical morbidities and complications over the last decade.

\section{Limited decompression and fusion}

a. Judgment depends on the degrees of instability versus the stability at the different curve segments.

b. Fusion with stabilization technique is superior to staged decompression and fusion at later stage

c. Solo decompression: is very frequently complicated by further collapse of the curve.

d. Appearance of severer type of pain after simple Laminectomy.

e. Another fusion surgery is required to treat that pain and iatrogenic instability.

f. Curve progression is very common if Decompression is done at the APEX of the curve without corrective fusion

g. Isolated Decompression at the bottom of rigid curve at L4-L5 and L5 -S1 (Transitional segment) may end up with imbalance situation with pain and curve progression.

\section{Curve correction procedures}

a. No correction is required in presence of balanced spine in frontal plane with sagittal alignment.

b. The presence of an old uncorrected adolescent rigid curve needs careful calculation before correction of degenerative Thoracolumbar and lumbar curves.

The rigid old curve cannot adapt to the corrected new position that will end up in chronic pain with global imbalance

\section{Flat back syndrome}

a. Loss of Lumbar Lordosis.

b. Need an extensive release of the posterior elements with facet joints osteotomy.

c. Anterior release MAY also be needed in severely rigid curves.

d. Anterior column reconstruction with cages especially at Lumbosacral junction is necessary and always required to restore sagittal balance (Figure 3). 


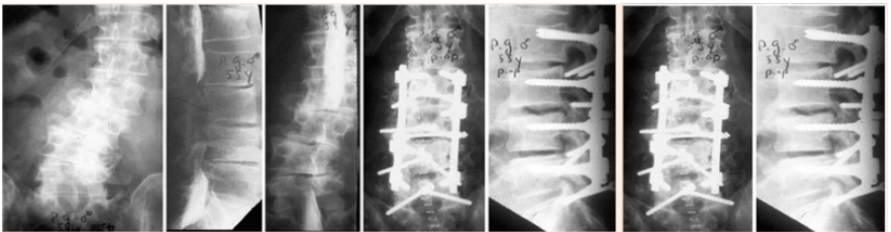

Figure 3 Flat back syndrome.

\section{Selective segmental osteotomies}

a. Mostly Trans pedicular resection osteotomies

b. Vertebral De- cancellation osteotomy

c. Wedging osteotomy for the associated kyphosis.

d. Total Vertebrectomy in one stage or two stages

e. Vertebral column resection for the associated kyphosis in particular, but not used with degenerative scoliosis so frequently.

f. These techniques became more frequently required for balance restoration in rigid and severely rigid curves of the thoracic and lumbar spine. ${ }^{1}$ (Figure $4 \mathrm{a} \& 4 \mathrm{~b}$ ).
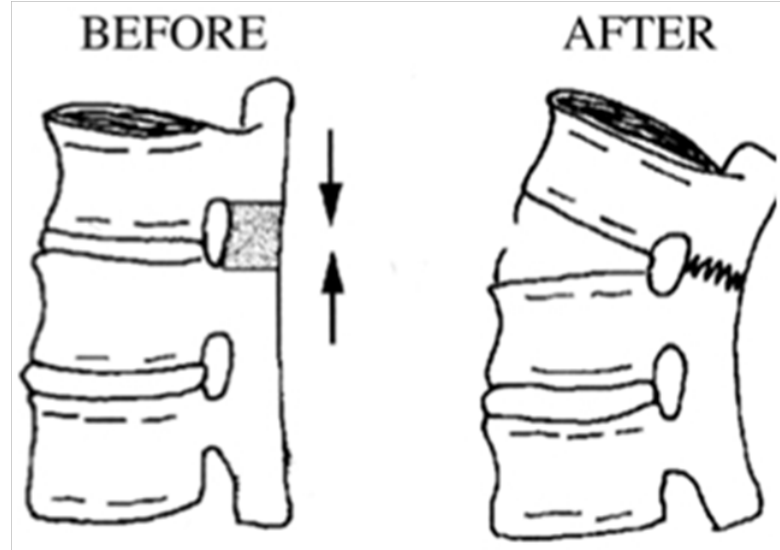

\section{Area of Bony Resection}

Figure 4a Smith Petersen Osteotomy.
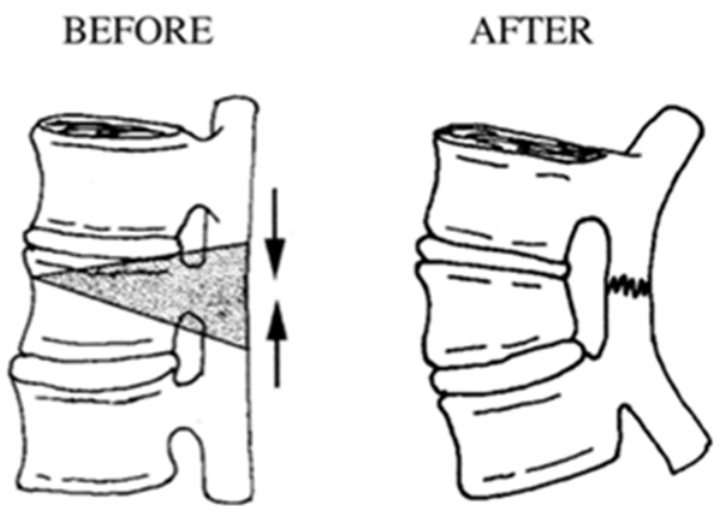

\section{Area of Bony Resection}

Figure 4b Three Column Pedicle Subtraction Osteotomy.
How to correct sagittal imbalance SPO vs PSO Osteotomy (Table I).

Table I Differences between SPO vs PSO.

\begin{tabular}{ll}
\hline Smith Petersen Osteotomy & $\begin{array}{l}\text { Three Column Pedicle } \\
\text { Subtraction Osteotomy }\end{array}$ \\
\hline $\begin{array}{l}\text { For the patient requiring } 10^{\circ} \text { to } 20^{\circ} \text { of } \\
\text { lordosis or } 4 \text { to } 7 \mathrm{~cm} \text { of correction of }\end{array}$ & $\begin{array}{l}\text { For the patient requiring } 20^{\circ} \text { to } 35^{\circ} \\
\text { of lordosis or } 10 \mathrm{~cm} \text { of correction } \\
\text { of the C7 plum line. }\end{array}$ \\
$\begin{array}{ll}\text { Needs a mobile disc. } & - \\
\text { The osteotomy hinges on the posterior } & \text { The osteotomy hinges on the } \\
\text { aspect of the disk } & \text { anterior cortex } \\
\text { Lengthens Ant Column. } & \text { Shortens post Column. }\end{array}$ \\
\hline
\end{tabular}

\section{Extended fixation and fusion}

a. Extended fusion to L5-S1.

b. It takes the movements off the lumbar spine.

c. It is the most critical segment to be considered whether or not to be included in fusion.

d. It is the most difficult fusion to be achieved.

e. 360 circumferential fusions at L5-S1 with anterior caging are the best to avoid nonunion.

f. Lumbosacral junction is usually degenerated.

\section{Stop of fusion at 15}

a. Put lot of stresses on lumbosacral junction.

b. Extension of fusion to the sacrum have an impact on the sacroiliac joints and hip joints

c. Facet joint artharosis at L5-S1 and transitional vertebral obliquity and rotation are quiet common.

d. Long whole spine $\mathrm{x}$ ray film is important to plane for the proper extension of the fusion for global correction.

e. Do not include extra motion segment in the fusion mass based on radiological finding only

f. Inclusion of Lumbosacral segment is questionable.

\section{Stopping the fixation and fusion at thoraco-} lumbar junction

a. Fixation to the D12 is not accepted as it is transitional site.

b. How to Avoid the Junctional Kyphosis.

c. Fixation should be extended toD9 or to D4 in some cases.

d. Global balance restoration is essential as the spine is stiff by degeneration not like adolescent spines that keeps resilient discs.

\section{Investigations}

\section{A. Plain $\mathrm{x}$ rays:}

B. Whole spine $\mathrm{x}$ ray films in coronal and sagittal planes.

C. Stress $x$ ray films -for the whole spine for mobility detection.

D. MRI Study for soft tissues \& spinal cord.

E. CT $-\&$ CT myelography in presence of sever kypho scoliosis.

F. Neurophysiological studies \& neuromonitoring.

G. Psychological assessment of elderly is very valuable before major surgery (Figure 5,6). 


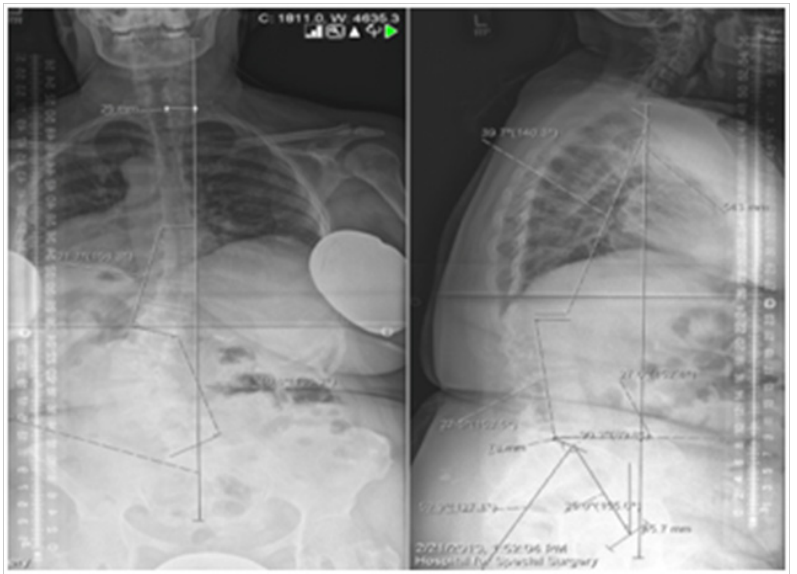

Figure 5 Whole spine $x$ ray films in coronal and sagittal planes.

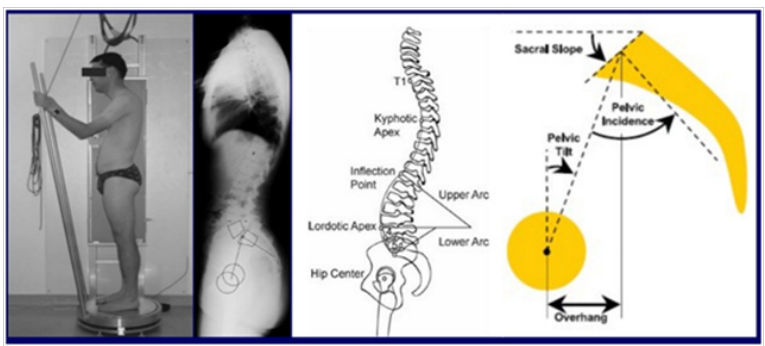

Figure 6 Spino-Pelvic alignment.

$\mathrm{PI}=\mathrm{PT}+\mathrm{SS}$

Pelvic incidence fixed anatomical value no matter what the position of the pelvis is

Angle between a line joining the center of the upper endplate of $\mathrm{s} 1$ to the axis of the femoral heads and a line perpendicular to the upper end plate of $\mathrm{s}^{2}{ }^{2}$ (Figure 7).

\section{Sacral slope}

Angle between the endplate of s1 and a horizontal line.

\section{Pelvic tilt}

Angle between a vertical line and the line joining the middle of the sacral plate and the axis of the femoral heads.

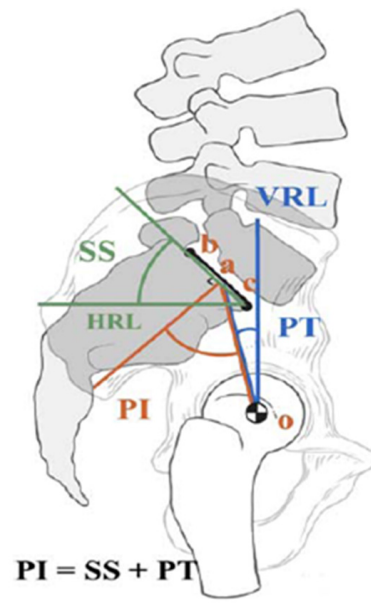

Figure 7 Spino pelvic alignment after surgical correction for developmental spondylolisthesis.

\section{Problems Encountered with Surgical Correction}

a. Length of fusion.

b. Inclusion of Lumbosacral junction.

c. Higher level of fusion.

d. Higher spinal segment adjacent to fusion and junctional kyphosis.

e. Previous scoliosis surgery.

f. One or multiple osteotomies for sagittal balance restoration.

g. General medical condition.

h. Osteoporosis Versus rigid instrumentations.

\section{Outcome and complications}

a. Most of complications are related to bad selection of patients for major surgical correction.

b. Wrong planning

c. Wrong surgical techniques \&approach related complications

d. Lack of balance at the final result after major surgery.

e. Implant failure

f. Patient satisfaction is linked with his muscle power

g. After surgery and preoperative professional activities

\section{Limited Intervention by Vertebral Augmentation and TLSO \& Medical TTT}

A. 76Years old lady attended with low and mid back pain over the last three months.

B. Has a history of L4-L5 cage fusion 15 years before.

C. Radiological examination shows:

i. Loss of Lumber Lordosis.

ii. High Degree of Dorso- Lumber kyphosis.

iii. Wedge compression fractures at D12 \&D11.

\section{Treatment:}

i. Vertebral Augmentation.

ii. TLOS Brace.

iii. Alendronate drugs for her osteoporosis (Figure 8).

\section{Global assessment of the different curves}

A. Whole Length Standing X rays films.

B. Proper Calculation of the dorsal kyphosis, the residual Lumbar Lordosis if still preserved.

C. Spino- Pelvic angles:

i. Sacral sloping angle.

ii. Pelvic tilt angle.

iii. Pelvic incidence angle.

D. $\mathrm{PI}=\mathrm{PT}+\mathrm{S} \mathrm{S}$

E. C2 to posterior upper corner of SI plum line either +ve or -ve Balance. 
F. The angle of correction should be equal to the PI ANGLE+9-10 DEGREES. That will restore the lost balance by restoration of normal Lordosis (50-60 Degrees) (Figure 9).
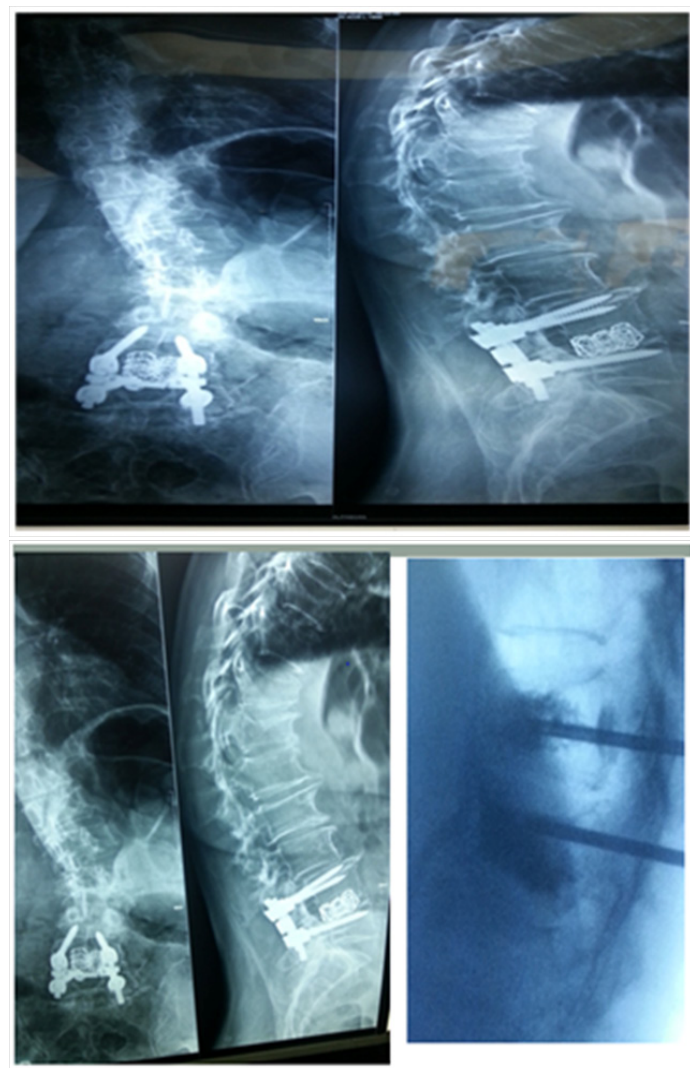

Figure 8 Limited intervention by vertebral augmentation andTLSO \& Medical ttt.
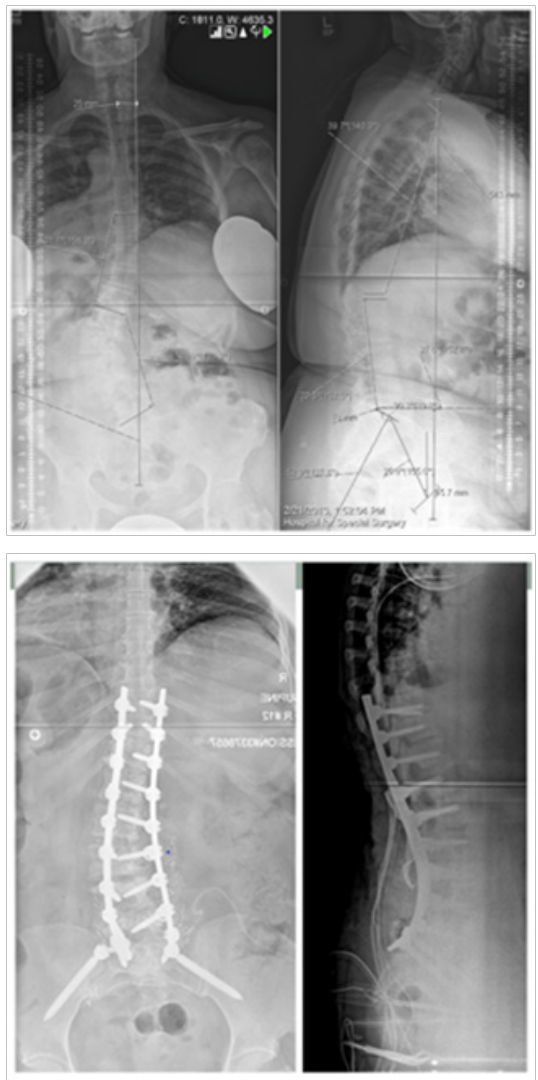
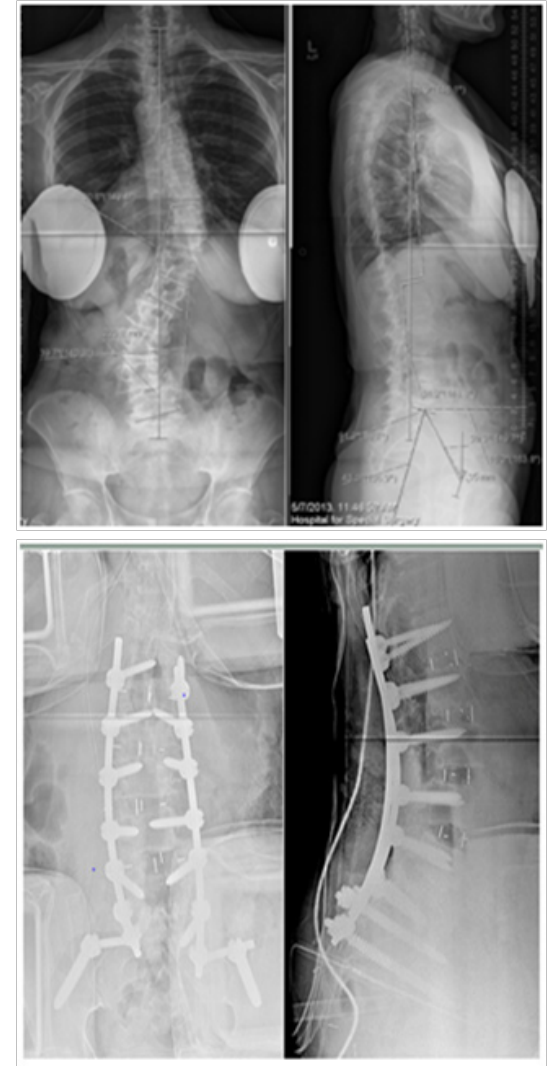

Figure 9 Global assessment of the different curves.

\section{Conclusion}

1. The relationship between clinical presentation and path morphology in Degenerative scoliosis still remaining as a challenge to spine surgeons.

2. Full analysis of the major signs and symptoms as instability, stenosis, unbalanced deformity and possible curve progression is very important before surgery.

3. Complex pain pattern Back pain, claudication, Sensory and motor weakness professional and activities affection should be analyzed and it's source's should be localized.

4. Correction of an exaggerated dorsal kyphosis is very important step; It should be done as a part of the corrective surgery in the same stage. If it is neglected the global balance could not be achieved.

5. Every patient problem should be individually analyzed to tailor out the required procedure.

6. Assessment of the results of surgical treatment through curve correction depend on

7. Certain and specific Modifiers

i. The degree of correction at the Apex of the curves whatever its site.

ii. Restoration of Lumber Lordosis

iii. Correction of Vertebral translation

iv. Spinal Balance restoration

v. Extended fusion to the sacrum 
8. Patient's satisfaction through changing of his quality of life by surgical intervention is the knee of success.

9. Sometimes it is not easy to gain the best result at such ages with multifactorial problems.

10. Muscle power restoration after surgery will improve the result and getting patients satisfaction.

\section{Limitations in egypt to reach optimum results}

a. Imaging \& soft ware systems to calculate angles.

b. Lack of proper setup and instrumentations.

c. No allograft availability.

d. BMP-Bone Morphogenic Protein.

e. Bone graft substitutes.

f. Neuro monitoring still not used routienly in our surgery.

g. Imuno- compromised patients at elder ages (liver-kidney).

h. Standard insurance system for all population.

i. Standard training programs \& specialized spine fellowship.,

\section{Acknowledgments}

None.

\section{Conflicts of interest}

None.

\section{References}

1. Bridwell KH, Lewis SJ, Edwards $\mathrm{C}$ et al. Complications and outcomes of pedicle subtraction osteotomies for fixed sagittal imbalance. Spine. 2003:28(18):2093-2101.

2. Labelle H, Roussouly P, Chopin D et al. Spino-pelvic alignment after surgical correction for developmental spondylolisthesis. Eur Spine J. 2008:17(9):1170-116

3. Aebi M The adult scoliosis. Eur Spine J. 2005:14(10):925-948.

4. Schwab F, Lafage V, Farcy JP et al. Surgical Rates and Operative Outcome Analysis in Thoracolumbar and Lumbar Major Adult Scoliosis: application of the New Adult Deformity Classification. Spine. 2007:32(24): 2723-2730. 